\title{
Perfil das condições de saúde das capitais brasileiras na perspectiva dos Objetivos de Desenvolvimento do Milênio (ODM)
}

Danielle Ramos de Miranda Pereira, Marcelo de Rezende Pinto, Alida Rosária Silva Ferreira e Rodrigo Nunes Ferreira

\section{Introdução}

Nos últimos anos, os administradores públicos têm considerado de substancial importância o estudo de indicadores sociais para balizar a formulação, o monitoramento e a avaliação das políticas públicas, inclusive das relacionadas às condições de saúde. Os sistemas de monitoramento e avaliação de políticas públicas contribuem para aperfeiçoar os mecanismos de controle da gestão, reduzindo a distância entre as informações dos cidadãos e do setor público, no que se refere aos resultados das políticas públicas (Cenneviva e Farah, 2006; ANDrade et al, 2001).

Nesse contexto, em que se tornou imprescindível o aperfeiçoamento de instrumentos de gestão pública e a ampliação da capacidade de controle da sociedade sobre as ações públicas, é crescente a demanda pelo uso de indicadores sociais com a finalidade de auxiliar a formulação de políticas públicas municipais em um contexto de descentralização administrativa e tributária a favor dos 
municípios e da institucionalização do processo de planejamento público local (Jannuzzi, 2001a, 2002; Guimarães e JANNUZZI, 2004).

Ademais, recentemente, o uso de indicadores sociais vem sendo amplamente divulgado e implementado para acompanhar a evolução dos países no que tange aos Objetivos de Desenvolvimento do Milênio (ODM) - projeto idealizado pela Organização das Nações Unidas (ONU) e assinado na Cúpula do Milênio, em 2000, por 189 países. As nações firmaram um pacto para tornar o mundo mais justo e solidário, até 2015 , por meio de oito iniciativas que ficariam conhecidas como os oito objetivos do milênio: 1) Erradicar a extrema pobreza e a fome; 2) Atingir o ensino básico universal; 3) Promover a igualdade entre os sexos e a autonomia das mulheres; 4) Reduzir a mortalidade infantil; 5) Melhorar a saúde materna; 6) Combater o HIV/Aids, a malária e outras doenças; 7) Garantir a sustentabilidade ambiental; e 8) Estabelecer uma parceria mundial para o desenvolvimento.

Para cada um desses oito objetivos foram estabelecidas metas a serem monitoradas e alcançadas até o ano de 2015:

- Objetivo 1: meta 1 - reduzir pela metade a proporção da população com renda inferior a 1 dólar por dia; e meta 2 - reduzir pela metade a proporção da população que sofre de fome;

- Objetivo 2: meta 3 - garantir que as crianças de ambos os sexos concluam o ensino elementar;

- Objetivo 3: meta 4 - eliminar a disparidade entre os sexos no ensino primário e secundário;

- Objetivo 4: meta 5 - reduzir em 2/3 a mortalidade de crianças menores de 5 anos;

- Objetivo 5: meta 6 - reduzir em 3/4 a taxa de mortalidade materna;
- Objetivo 6: meta 7 - ter detido e começado a reverter a propagação do HIV/Aids; e meta 8 - ter detido e começado a reverter a propagação da malária e outras doenças;

- Objetivo 7: meta 9 - integrar os princípios de desenvolvimento sustentável nas políticas e programas nacionais e reverter a perda de recursos ambientais; meta 10 - reduzir pela metade a proporção da população sem acesso permanente e sustentável à água potável e ao esgotamento sanitário; e meta 11 - alcançar uma melhoria significativa na vida de pelo menos 100 milhões de habitantes de assentamentos precários;

- Objetivo 8: meta 12 - avançar no desenvolvimento de um sistema comercial e financeiro aberto, baseado em regras, previsível e não discriminatório; meta 13 atender às necessidades dos países menos desenvolvidos, incluindo um regime não sujeito a cotas para as exportações desses países e uma ajuda pública mais generosa para o desenvolvimento dos países empenhados na luta contra a pobreza; meta 14 - atender às necessidades especiais dos países sem acesso ao mar e dos pequenos Estados insulares em desenvolvimento; meta 15 - tratar globalmente o problema da dívida dos países em desenvolvimento, mediante medidas nacionais e internacionais de modo a tornar a sua dívida sustentável; meta 16 - em cooperação com os países em desenvolvimento, formular e executar estratégias que permitam aos jovens obter trabalho digno e produtivo; meta 17 - em cooperação com as empresas farmacêuticas, proporcionar o acesso a medicamentos essenciais a preços acessíveis nos países em desenvolvimento; e meta 18 - em cooperação com o setor privado, tornar acessíveis os benefícios de novas tecnologias, em especial das tecnologias de informação e de comunicação. 
Sendo assim, surgiu o interesse de investigar nas capitais brasileiras a situação da mortalidade na infância (relacionada ao objetivo 4 e meta 5); da saúde materna (objetivo 5 e meta 6); da propagação do HIV/Aids, da malária e outras doenças (objetivo 6 e metas 7 e 8); e do saneamento (objetivo 7 e meta 10). Este artigo será, então, focado nas metas do milênio relacionadas diretamente à área da saúde e ao saneamento.

A partir dos indicadores sugeridos pela ONU (2003) para o monitoramento e a avaliação do cumprimento dessas metas, o presente artigo tem por objetivo delinear os perfis das capitais brasileiras quanto às condições de saúde, a fim de contribuir para a formulação e avaliação de políticas públicas e indicar um caminho para a escolha de indicadores importantes a serem monitorados. A justificativa deste trabalho reside na constatação da existência de poucos estudos, no contexto da administração pública no Brasil, sobre a utilização das metas estabelecidas pelos ODM para subsidiar a formulação e avaliação das políticas públicas. Dessa forma, o artigo pretende contribuir com a discussão das questões que envolvem a temática.

Este artigo está estruturado da seguinte forma: de início, faz considerações sobre os indicadores sociais quanto à definição, às principais limitações, propriedades desejáveis e tipologias de classificação utilizadas na formulação e na avaliação de políticas públicas. Em seguida, são apresentados os dados, os procedimentos metodológicos adotados no estudo e a análise dos resultados obtidos com a adoção do método de Grade of Membership (GoM). Por fim, são discutidas as conclusões e considerações gerais do estudo.

\section{O uso de indicadores sociais na formulação e na avaliação de políticas públicas}

O surgimento dos indicadores sociais está diretamente relacionado à consolidação do planejamento no setor público ao longo do século XX. No entanto, o desenvolvimento de um marco conceitual sobre os indicadores sociais ganhou corpo científico em meados dos anos 1960, no bojo das

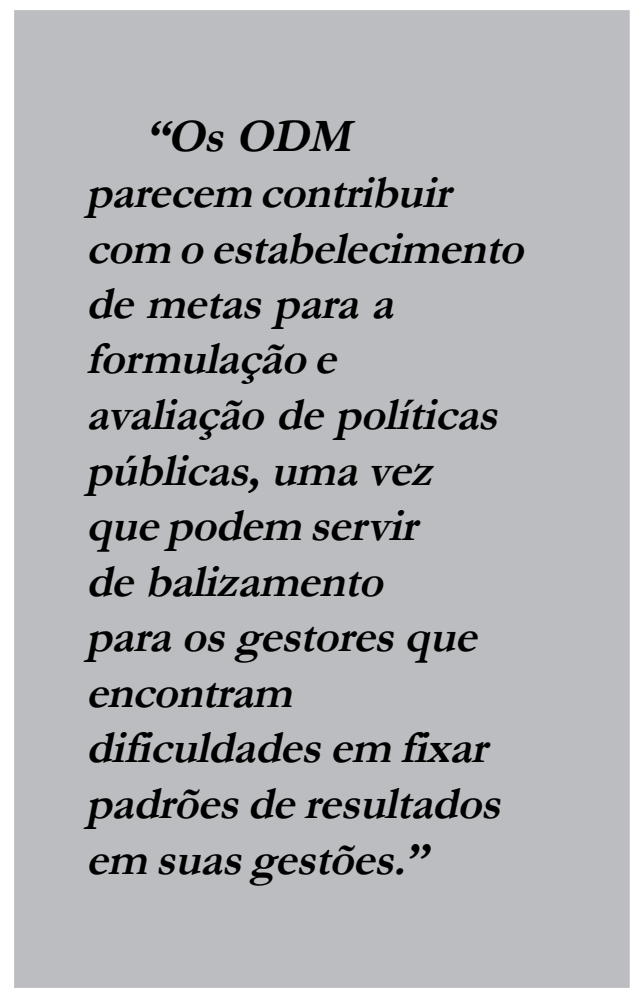

tentativas de organização de sistemas mais abrangentes de acompanhamento das transformações sociais e aferição do impacto das políticas sociais nas sociedades desenvolvidas e subdesenvolvidas" (JAnnuzzI, 2002).

Nessa época, o desenvolvimento era medido por meio do PIB per capita, que não levava em consideração questões importantes para medir a qualidade de 
vida, mas apenas o aspecto econômico da população (Torres et al, 2003). Com a criação do Índice de Desenvolvimento Humano (IDH), na década de 1990, foi possível sintetizar as dimensões renda, longevidade e escolaridade, captando melhor os diversos aspectos da população. A partir daí, outros indicadores de abrangência nacional, estadual e municipal começaram a surgir para responder às mais variadas questões (PNUD, 1998).

Diante das grandes diferenças regionais existentes no Brasil, é preciso conhecer muito bem o universo para o qual a política será direcionada. Nos últimos anos, vem-se investindo em novas técnicas para organizar as informações coletadas e criar indicadores que sejam capazes de retratar melhor o cenário onde as políticas sociais irão atuar. No entanto, erros ainda são cometidos ao se analisar determinados indicadores, porque é necessário um diagnóstico prévio do sistema em que a política avaliada está inserida para melhor visualizar todo o processo, que vai da implementação da política até seu resultado final (Silva e Melo, 2000).

Muitas vezes espera-se que os indicadores sociais, por si só, tragam uma resposta efetiva para a aplicação de recursos, mas isso, na realidade, não é verdadeiro (JANNUZZI, 2002). Entretanto, conforme alerta o autor, é preciso considerar os limites na utilização dos indicadores sociais, uma vez que não são substitutos dos conceitos dos quais se originam, mas instrumentos metodológicos que contribuem para a percepção empírica dos conceitos, permitindo o aprofundamento da investigação acadêmica sobre a mudança social e viabilizando, no campo das políticas públicas, a avaliação das ações e programas sobre a realidade social.
Ademais, conforme a Organização Mundial de Saúde (OMS, 1996), todo indicador deve possuir quatro características científicas desejáveis: validade, isto é, a capacidade de representar o fenômeno que pretende medir; confiabilidade, a capacidade de fornecer os mesmos resultados quando calculados em circunstâncias similares; sensibilidade, a capacidade de refletir mudanças no fenômeno de interesse; e especificidade, refletir mudanças em fenômenos específicos de interesse. Além dessas quatro características científicas, em uma situação ideal, o indicador social deve possuir relevância para a discussão da agenda da política social; grau de cobertura populacional adequado; simplicidade para o entendimento dos agentes das políticas e do público-alvo dessas políticas; atualização periódica; desagregação em termos socioeconômicos e demográficos; e uma série histórica.

Segundo Arretche (1998) e Andrade et al (2001), outra tipologia que tem sido muito adotada para a classificação dos indicadores sociais na formulação e na avaliação de políticas sociais é a que os diferencia em função das três dimensões da avaliação de políticas sociais: a eficiência, a eficácia e a efetividade. A eficiência deve ser entendida como a relação entre o esforço (meios e recursos) empregado na implementação de uma política e os seus resultados. Por sua vez, a eficácia se refere à relação entre os objetivos e instrumentos utilizados e seus resultados efetivos, enquanto a efetividade é o exame da relação entre a implementação e os impactos na mudança de uma condição social prévia (Arretche, 1998; Andrade et al, 2001).

O Quadro 1 ilustra a distinção entre as três dimensões da avaliação e os recursos analíticos utilizados em cada um dos aspectos da avaliação. 
Quadro 1: As dimensões da avaliação de políticas públicas

\begin{tabular}{|c|l|l|l|}
\hline Dimensão & \multicolumn{1}{|c|}{ Conceito } & \multicolumn{1}{c|}{ Objetivo } & \multicolumn{1}{c|}{ Relação } \\
\hline Eficiência & $\begin{array}{l}\text { Relação entre os esforços } \\
\text { empregados em uma ação e os } \\
\text { resultados alcançados. }\end{array}$ & $\begin{array}{l}\text { Verificar aspectos referentes } \\
\text { ao rendimento técnico e } \\
\text { administrativo da ação. }\end{array}$ & $\begin{array}{l}\text { Custo/Benefício } \\
\text { (preços de mercado) }\end{array}$ \\
\hline Eficácia & $\begin{array}{l}\text { Relação entre objetivos e } \\
\text { instrumentos utilizados e } \\
\text { os resultados efetivos. }\end{array}$ & $\begin{array}{l}\text { Verificar a penetração de } \\
\text { resultados no tempo e no } \\
\text { espaço. }\end{array}$ & $\begin{array}{l}\text { Metas enunciadas/ } \\
\text { Metas alcançadas } \\
\text { (preços sociais). }\end{array}$ \\
\hline Efetividade & $\begin{array}{l}\text { Relação entre a implementação } \\
\text { e os seus impactos na mudança } \\
\text { de uma condição prévia. }\end{array}$ & $\begin{array}{l}\text { Verificar os efeitos em } \\
\text { cadeia dentro da sociedade. }\end{array}$ & $\begin{array}{l}\text { Não se ocupa de inputs e } \\
\text { outputs como as dimensões } \\
\text { anteriores. } \\
\text { Refere-se a impacto, isto é, } \\
\text { aos valores sociais. }\end{array}$ \\
\hline
\end{tabular}

Fonte: Andrade et al (2001)

Quanto às três dimensões da avaliação das políticas sociais, os indicadores são classificados em: de eficiência dos meios e recursos empregados; da eficácia no cumprimento das metas; e da efetividade social do programa, ou seja, dos impactos do programa em termos de melhorias no bem-estar social.

\section{Dados e metodologia}

\section{Dados}

Neste tópico são descritos os indicadores sugeridos pela ONU (2003) para o monitoramento das metas dos ODM diretamente ligadas à saúde e ao saneamento. Os principais indicadores selecionados para acompanhar a evolução da meta 5 , referente à redução da mortalidade na infância, são a taxa de mortalidade infantil e a taxa de mortalidade na infância em menores de cinco anos. A taxa de mortalidade infantil é definida como a razão entre o número de óbitos de menores de um ano de idade e o total de nascidos vivos em uma região geográfica, em determinado ano. De acordo com os critérios da Organização Mundial de Saúde (OMS), costuma-se classificar a taxa de mortalidade infantil como baixa, se for menor que 20; média, entre 20 e 49; e alta, a partir de 50 óbitos por mil nascidos vivos (Pereira, 1995; Unicef, 2008). A taxa de mortalidade na infância corresponde à razão entre o número de óbitos de menores de cinco anos de idade, de determinada região em um período específico, e a população de crianças na faixa etária correspondente. Em 2005, a taxa de mortalidade na infância foi de 31,1 óbitos por mil nascidos vivos no Brasil (UNICEF, 2008).

Para acompanhar a evolução da meta 6 , relacionada à melhoria da saúde materna, a ONU (2003) seleciona como indicadores a razão da mortalidade materna e a proporção de partos realizados por profissionais de saúde. A razão da mortalidade materna equivale à divisão entre o número de óbitos maternos e o total de nascidos vivos em uma região geográfica, em um determinado ano. O índice aceitável pela Organização Mundial da Saúde para a razão da mortalidade materna é de 20 mortes maternas por 10 mil nascidos vivos (UNICEF, 2008).

Os relatórios nacionais de acompanhamento dos ODM elaborados pelo Ipea (2005, 2007) têm incluído ainda, como indicador da saúde materna, a 
proporção de gestantes que realizaram sete ou mais consultas no pré-natal. No Brasil, a proporção de gestantes que realizaram sete ou mais consultas no pré-natal, em 2004, foi de 52,93\% (Datasus, 2008). No relatório "Situação Mundial da Infância 2008”, a proporção de partos cesarianos, que conforme a OMS não deve ultrapassar os 15\%, também é considerada no contexto da saúde materna (Unicef, 2008).

Entre os principais indicadores sugeridos pela ONU (2003) para monitorar a propagação do HIV/Aids (meta 7 ) está a prevalência do HIV entre as gestantes de 15 a 24 anos, ou seja, a proporção de gestantes cujo resultado do exame de HIV/Aids foi positivo. Nos relatórios nacionais de acompanhamento dos objetivos do milênio, a taxa de incidência de HIV/Aids também figurou entre os indicadores que monitoravam a propagação da doença (Ipea, 2005, 2007). Essa taxa é definida como a razão entre o número de casos novos confirmados da síndrome da imunodeficiência adquirida e a população total de uma região geográfica em determinado período. Em 2005, a incidência de HIV/Aids no Brasil foi de 15,05 por 100 mil habitantes (Datasus, 2008). Reduções nas taxas de incidência dessa doença, observadas nos últimos anos, resultam, em parte, do atraso na notificação dos dados, fazendo com que seja necessária muita cautela na análise dos dados (Opas, 2008).

Com relação à meta 8 , que propõe a redução da propagação da malária e outras doenças, a ONU (2003) tem sugerido acompanhar, entre outras doenças, a situação da tuberculose, considerando as taxas de mortalidade e de prevalência e a proporção de casos detectados e curados. As taxas de prevalência e de mortalidade de tuberculose referem-se, respectivamente, ao número de casos confirmados e ao número de mortes causadas pela doença por habitante de uma região geográfica em determinado ano. No Brasil, a taxa de mortalidade de tuberculose respiratória, em 2005, foi de 2,32 por 100 mil, calculada de acordo com os dados disponíveis em Datasus (2008). Para o percentual de cura da doença, a meta mundial pactuada junto à OMS é de no mínimo 85\% (MinistÉRIO DA SAÚde, 2008). Considerando que se espera um percentual de cura de aproximadamente 100\%, quando não se abandona o tratamento, foi adotado o limite de $15 \%$ para a proporção de abandono do tratamento da tuberculose. Essa proporção é calculada pela razão entre o número de casos novos encerrados por abandono e o número de casos diagnosticados (Datasus, 2008).

Os principais indicadores para o monitoramento da meta 10 , referente à redução da proporção de pessoas sem acesso permanente e sustentável à água potável e ao esgotamento sanitário, são: as proporções da população com acesso à água tratada e a melhores condições de esgotamento sanitário, incluindo as condições que não permitem contato humano ou animal com resíduos humanos excretados. Esses indicadores foram contemplados no estudo, principalmente em virtude da relação entre eles e a mortalidade infantil. De acordo com o Ipea (2005), em 2003 a proporção de moradores de domicílios particulares permanentes com abastecimento de água por rede geral no Brasil foi de 91,4\%. Contudo, no mesmo ano, apenas 54,3\% dos moradores de domicílios particulares permanentes no Brasil possuíam acesso à rede coletora de esgoto.

A seleção dos indicadores utilizados na definição dos perfis das condições de saúde 
das capitais brasileiras foi baseada no conjunto dos principais indicadores sugeridos pela ONU, cujos dados estavam disponíveis em Datasus (2008) e SNIS (2008). Os valores dos indicadores selecionados para a definição dos perfis foram categorizados considerando-se, em geral, os limites estabelecidos pela Organização Mundial de Saúde ou os valores referentes ao Brasil em 2005.

O Quadro 2 contém os indicadores selecionados por meta, a fonte dos dados e os limites estabelecidos para a categorização dos valores, com base na bibliografia sobre o tema. Esses indicadores estão divididos em indicadores-processo (proporção de gestantes que realizaram sete ou mais consultas no pré-natal, proporção de partos cesarianos, proporção de abandono no tratamento da tuberculose, proporção da população com acesso à rede geral de água, proporção da população com acesso à rede geral de esgotamento sanitário) e indicadoresproduto (taxa de mortalidade infantil, taxa de mortalidade na infância, razão da mortalidade materna, taxa de incidência de HIV/Aids, taxa de mortalidade por tuberculose respiratória) para a formulação e/ou avaliação de políticas públicas de saúde.

\section{O Método de Grade of Membership}

Para o delineamento de perfis das capitais brasileiras quanto às condições de saúde, de acordo com a perspectiva dos

Quadro 2: Indicadores selecionados por meta e os limites estabelecidos para a categorização dos seus valores

\begin{tabular}{|c|c|c|c|}
\hline Meta & Indicador & Fonte dos Dados & Categorias \\
\hline \multirow[t]{2}{*}{ Meta 5} & $\begin{array}{l}\text { Taxa de mortalidade infantil } \\
\text { por mil }\end{array}$ & $\begin{array}{l}\text { Calculada de acordo com os dados } \\
\text { disponíveis em Estatísticas Vitais } \\
\text { (DATASUS, 2008) }\end{array}$ & $\begin{array}{l}\text { 1) } \geqslant 50 \\
\text { 2) de } 20 \text { a } 49 \\
\text { 3) }<20\end{array}$ \\
\hline & $\begin{array}{l}\text { Taxa de mortalidade na infância } \\
\text { por mil }\end{array}$ & $\begin{array}{l}\text { Calculada de acordo com os dados } \\
\text { disponíveis em Informações de Saúde } \\
\text { (DATASUS, 2008) }\end{array}$ & $\begin{array}{l}\text { 1) }>31,1 \\
\text { 2) } \leqslant 31,1\end{array}$ \\
\hline \multirow{3}{*}{ Meta 6} & $\begin{array}{l}\text { Razão da mortalidade materna } \\
\text { por } 10 \mathrm{mil}\end{array}$ & $\begin{array}{l}\text { Calculada de acordo com os dados } \\
\text { disponíveis em Estatísticas Vitais } \\
\text { (DATASUS, 2008) }\end{array}$ & $\begin{array}{l}\text { 1) }>20 \\
\text { 2) } \leqslant 20\end{array}$ \\
\hline & $\begin{array}{l}\text { Proporção de gestantes que } \\
\text { realizaram } 7 \text { ou mais consultas } \\
\text { no pré-natal }\end{array}$ & $\begin{array}{l}\text { Disponível em Indicadores e dados } \\
\text { básicos (DATASUS, 2008) }\end{array}$ & $\begin{array}{l}\text { 1) }<52,93 \% \\
2) \geqslant 52,93 \%\end{array}$ \\
\hline & Proporção de partos cesarianos & $\begin{array}{l}\text { Disponível em Indicadores do Pacto de } \\
\text { Atenção Básica (DATASUS, 2008) }\end{array}$ & $\begin{array}{l}\text { 1) }>15 \% \\
\text { 2) } \leqslant 15 \%\end{array}$ \\
\hline Meta 7 & $\begin{array}{l}\text { Taxa de incidência de Aids } \\
\text { por } 100 \text { mil }\end{array}$ & $\begin{array}{l}\text { Disponível em Indicadores e dados } \\
\text { básicos (DATASUS, 2008) }\end{array}$ & $\begin{array}{l}\text { 1) }>15,05 \\
\text { 2) } \leqslant 15,05\end{array}$ \\
\hline \multirow[t]{2}{*}{ Meta 8} & $\begin{array}{l}\text { Taxa de mortalidade por } \\
\text { tuberculose respiratória por } \\
100 \text { mil }\end{array}$ & $\begin{array}{l}\text { Calculada de acordo com os dados } \\
\text { disponíveis em Informações de Saúde } \\
\text { (DATASUS, 2008) }\end{array}$ & $\begin{array}{l}\text { 1) }>2,32 \\
\text { 2) } \leqslant 2,32\end{array}$ \\
\hline & $\begin{array}{l}\text { Proporção de abandono no } \\
\text { tratamento da tuberculose }\end{array}$ & $\begin{array}{l}\text { Disponível em Indicadores do Pacto de } \\
\text { Atenção Básica (DATASUS, 2008) }\end{array}$ & $\begin{array}{l}\text { 1) }>15 \% \\
\text { 2) } \leqslant 15 \%\end{array}$ \\
\hline \multirow[b]{2}{*}{ Meta 10} & $\begin{array}{l}\text { Proporção da população com } \\
\text { acesso à rede geral de água }\end{array}$ & $\begin{array}{l}\text { Disponível em Água e Esgoto } \\
\text { (SNIS, 2008) }\end{array}$ & $\begin{array}{l}\text { 1) }<91,4 \% \\
\text { 2) } \geqslant 91,4 \%\end{array}$ \\
\hline & $\begin{array}{l}\text { Proporção da população com } \\
\text { acesso à rede geral de esgotamento } \\
\text { sanitário }\end{array}$ & $\begin{array}{l}\text { Disponível em Água e Esgoto } \\
\text { (SNIS, 2008) }\end{array}$ & $\begin{array}{l}\text { 1) }<53,4 \% \\
\text { 2) de } 53,4 \% \text { a } \\
\text { a } 89,9 \% \\
\text { 3) } \geqslant 90 \%\end{array}$ \\
\hline
\end{tabular}

Fonte: Datasus (2008) e SNIS (2008) 
Objetivos de Desenvolvimento do Milênio, adotou-se o método de Grade of Membership (GoM). Este utiliza a Teoria de Conjuntos Nebulosos (Fuzzys), baseada no conceito dos subconjuntos imprecisos, que decorre da constatação de que muitas vezes as classes de objetos encontrados na natureza não possuem critérios de participação claramente definidos.

Além do método de GoM possibilitar a análise de dados categóricos de alta dimensão, segundo Machado (1997), esse método, quando comparado a outras técnicas disponíveis de análise de clustering, apresenta, entre outras, a vantagem de analisar pequenas amostras com grande número de variáveis. $\mathrm{O}$ método também permite revelar, de forma muito simples, a heterogeneidade presente nos dados, uma vez que o grau de pertinência de cada cidade é dado pela conjunção, nessa capital, de todas as categorias das variáveis do modelo (SAWYER et al, 2002).

A técnica de GoM estima, com base em um modelo multinomial, dois parâmetros: a probabilidade de uma categoria $l$, de uma variável $j$, pertencer ao perfil extremo $k, \lambda_{k j l}$ e, também, o grau de pertinência $g_{i k}$, de uma cidade $i$, a um perfil extremo $k$.

Como esses parâmetros são estimados de forma interativa, pode-se interpretar que $\lambda_{k j,}$, por meio do qual se caracterizam os perfis extremos, mede a probabilidade de que se tenha uma capital, com grau de pertinência total ao perfil $k$, dado o valor da categoria $l$, da variável $j$, enquanto que $g_{i k}$ representa o grau de proximidade que cada cidade tem ao perfil extremo $k$.

O grau de pertinência $g_{i k}$ é medido em função da comparação das possíveis combinações de valores nas categorias $l$ da cidade com o conjunto de valores nas categorias $l$, configurados no perfil extremo. Para cada cidade, em um conjunto nebuloso, existe um escore de grau de pertinência $\left(g_{i k}\right)$ que representa o grau com que uma cidade $i$ pertence ao perfil extremo k. Esses escores podem variar no intervalo [0,1]. O zero indica que a cidade não pertence ao conjunto e o um, que a cidade pertence completamente ao conjunto.

Assim, o valor de $g_{i \text {. }}$ representa a proporção ou intensidade de pertinência a cada perfil extremo, de forma que se tem as seguintes restrições para a medida (SAWYER et al, 2000):

$$
\begin{aligned}
& g_{i k} \geq 0 \text { para cada } i \mathrm{e} j . \\
& \sum_{k=1}^{K} g_{i k}=1 \text { para cada } i .
\end{aligned}
$$

Baseando-se nas considerações de Manton et al (1994) e de Sawyer et al (2000), para a estimação dos parâmetros do modelo, a fim de delinear o perfil das capitais, são necessários os seguintes pressupostos:

1) As variáveis aleatórias $Y_{i j l}-$ em que $i$ se refere à capital, $j$ à variável (ou indicador) e $l$ à categoria da variável $j$ - são independentes para diferentes valores de $i$. Ou seja, os valores dos indicadores das capitais são independentes;

2) Os $g_{i k}(k=1,2, \ldots K)$ são realizações das componentes do vetor aleatório $\xi_{i}=\left(\xi_{i \uparrow} \ldots \xi_{i k}\right)$ com função de distribuição $H(x)=\operatorname{Pr}\left(\xi_{i}<=x\right)$.

3) Se o grau de pertinência $g_{i k}$ for conhecido para a capital $i$, os valores dos indicadores $\left(Y_{i j l}\right)$, em suas categorias, são independentes;

4) A probabilidade de valor $l$, para o jésimo indicador, da capital com k-ésimo perfil extremo é $\lambda_{k j l}$. Como pressuposto do modelo, existe pelo menos uma capital que é um membro bem definido de 
k-ésimo perfil. Esse pressuposto dá a probabilidade de determinado valor, em uma capital, para as categorias de cada indicador. Matematicamente, o pressuposto pode ser escrito da seguinte forma:

$$
\begin{aligned}
& \lambda_{k j l} \geq 0 \text { para cada k, j, l. } \\
& \sum_{i=1}^{L_{i}} \lambda_{k j l}=1 \text { para cada k e j. }
\end{aligned}
$$

5) A probabilidade de um valor de nível $l$, do j-ésimo indicador, pela capital $i$, condicionada ao escore $g_{i k}$, será dada por:

$$
\operatorname{Pr}\left(Y_{i j l}=1\right)=\sum_{k=1}^{K} g_{i k} \lambda_{k j l}
$$

De acordo com os pressupostos acima, o modelo de máxima verossimilhança pode ser dado pela seguinte equação:

$$
L(y)=\prod_{i=1}^{l} \prod_{j=1}^{J} \prod_{l=1}^{L j}\left(\sum g_{i k} \lambda_{k j l}\right)^{y_{j l l}}
$$

O software GoM foi utilizado para estimação dos parâmetros do modelo, possibilitando a caracterização dos perfis das capitais quanto às condições de saúde, sob a perspectiva dos Objetivos de Desenvolvimento do Milênio, assim como o conhecimento do grau de pertinência de todas as capitais brasileiras em relação aos perfis caracterizados.

\section{Análise dos resultados}

Número e delineamento de perfis

Como não existe procedimentopadrão para determinar a quantidade de perfis, a decisão do número de perfis utilizados depende de regularidades empíricas nos dados, que podem ser reproduzidas com alguma fidelidade por um modelo (BERKMAN et al, 1989), e do objetivo pretendido.

Uma vez que o método de GoM possibilita a obtenção do número desejado de perfis, inicialmente foram realizadas aplicações com dois e três perfis. A aplicação do modelo com dois perfis foi considerada mais satisfatória para o delineamento dos perfis das capitais quanto às condições de saúde, conforme explicado a seguir.

Os indicadores que foram selecionados para o estudo, mas não contribuíram para o delineamento do perfil, porque apresentaram apenas uma categoria nas diversas capitais, foram: a taxa de mortalidade na infância, a razão de mortalidade materna, a proporção de partos cesarianos e a proporção de abandono no tratamento da tuberculose. Com relação à taxa de mortalidade na infância em 2005, todas as capitais brasileiras apresentaram valores menores ou iguais a 31,1 óbitos de crianças entre 0 e 5 anos de idade por mil nascidos vivos. No mesmo período, as razões de mortalidade materna nas capitais foram menores ou iguais ao limite máximo aceitável pela Organização Mundial da Saúde (OMS), que é de no máximo 20 mortes maternas por 10 mil nascidos vivos (Unicef, 2008). Entretanto, a proporção de partos cesarianos em todas as capitais excedeu o limite máximo de $15 \%$, preconizado pela OMS (Unicef, 2008). Em relação ao tratamento da tuberculose, os resultados das capitais brasileiras foram positivos. Considerando que se espera percentual de cura próximo a 100\% quando não se abandona o tratamento, a proporção de abandono, em todas as capitais, foi menor ou igual ao limite máximo de $15 \%$, adotado mediante a meta mundial pactuada junto à OMS de curar 85\% dos casos de tuberculose (Opas, 2008).

Assim sendo, dos dez indicadores selecionados de acordo com as metas de saúde e saneamento dos Objetivos de Desenvolvimento do Milênio, seis contribuíram para o delineamento dos perfis das 
condições de saúde das capitais brasileiras em 2005.

A Tabela 1 apresenta as frequências absoluta e relativa das capitais, conforme as categorias dos indicadores e as estimativas de $\lambda_{k j l}$ para os dois perfis extremos considerados. As características de cada um desses perfis foram obtidas comparando $\lambda_{k j l} \mathrm{com}$ a frequência relativa das respostas na mesma categoria. Se $\lambda_{k j l}$ for significativamente maior, representa que a estimativa da probabilidade de uma capital, que pertence ao perfil $k$, ter o valor para um indicador em uma determinada categoria é significativamente maior que a estimativa da probabilidade marginal e, então, considera-se que essa categoria seja característica de capitais do perfil $k$.

Embora os perfis possam ser determinados com base em critérios técnicos, nesse estudo eles foram definidos a partir da avaliação da 'significância substantiva' dos perfis extremos, ou seja, a cada perfil novo criado procurou-se verificar se as mudanças observadas podiam ser justificadas do ponto de vista teórico (SAWYER et al, 2002, p. 760).

Dessa forma, considerou-se que uma categoria $l$, de uma variável $j$, seria característica de um perfil $k$ se a relação $\lambda_{k j l}$ e a frequência marginal fossem iguais ou maiores que 1,15. Esse valor arbitrário parece captar bem as características dominantes dos perfis extremos.

Os valores de $\lambda_{k j l}$ que preenchem as condições estabelecidas no parágrafo anterior foram destacados. Assim, os dois perfis extremos das capitais brasileiras, quanto às condições de saúde de acordo com a perspectiva dos Objetivos de Desenvolvimento do Milênio, foram delineados, segundo suas características, da seguinte forma:

a) Perfil extremo 1 (melhores condições de saúde de acordo com a perspectiva dos ODM)

É o perfil da capital que possui:

- Taxas intermediárias de mortalidade infantil;

Tabela 1: Estimativas de $\lambda_{k j l}$ segundo os perfis extremos, as categorias dos indicadores e as respectivas frequências marginais

\begin{tabular}{|c|c|c|c|c|c|c|}
\hline \multirow[t]{2}{*}{ Meta } & \multirow[t]{2}{*}{ Indicador } & \multirow[t]{2}{*}{ Categoria } & \multicolumn{2}{|c|}{$\begin{array}{c}\text { Frequência } \\
\text { Marginal }\end{array}$} & \multicolumn{2}{|c|}{$\begin{array}{l}\text { Perfis } \\
\text { Extremos }\end{array}$} \\
\hline & & & Absoluta & Relativa & 1 & 2 \\
\hline \multirow{5}{*}{ Meta 5} & \multirow{3}{*}{ Taxa de mortalidade infantil por mil } & 1 & 0 & 0,00 & 0,00 & 0,00 \\
\hline & & 2 & 8 & 0,30 & 0,00 & 3,38 \\
\hline & & 3 & 19 & 0,70 & 1,42 & 0,00 \\
\hline & \multirow{2}{*}{$\begin{array}{l}\text { Proporção de gestantes que realizaram } \\
\text { sete ou mais consultas no pré-natal }\end{array}$} & 1 & 13 & 0,48 & 0,00 & 2,08 \\
\hline & & 2 & 14 & 0,52 & 1,93 & 0,00 \\
\hline \multirow{2}{*}{ Meta 7} & \multirow{2}{*}{ Taxa de incidência de Aids por 100 mil } & 1 & 21 & 0,78 & 1,29 & 0,45 \\
\hline & & 2 & 6 & 0,22 & 0,00 & 2,93 \\
\hline \multirow{2}{*}{ Meta 8} & \multirow{2}{*}{$\begin{array}{l}\text { Taxa de mortalidade por tuberculose } \\
\text { respiratória por } 100 \mathrm{mil}\end{array}$} & 1 & 13 & 0,48 & 0,83 & 1,24 \\
\hline & & 2 & 14 & 0,52 & 1,15 & 0,78 \\
\hline \multirow{5}{*}{$\begin{array}{c}\text { Meta } \\
10\end{array}$} & \multirow{2}{*}{$\begin{array}{l}\text { Proporção da população com acesso à } \\
\text { rede geral de água }\end{array}$} & 1 & 6 & 0,22 & 0,00 & 3,72 \\
\hline & & 2 & 21 & 0,78 & 1,29 & 0,22 \\
\hline & \multirow{3}{*}{$\begin{array}{l}\text { Proporção da população com acesso à } \\
\text { rede geral de esgotamento sanitário }\end{array}$} & 1 & 17 & 0,63 & 0,58 & 1,59 \\
\hline & & 2 & 8 & 0,30 & 1,73 & 0,00 \\
\hline & & 3 & 2 & 0,07 & 1,64 & 0,00 \\
\hline
\end{tabular}

Fonte: Elaborada pelos autores 
- Alta proporção de gestantes que realizaram sete ou mais consultas no pré-natal;

- Alta taxa de incidência de HIV / Aids;

- Baixa taxa de mortalidade por tuberculose respiratória;

- Média e alta proporção da população com acesso à rede geral de água;

- Alta proporção da população com acesso à rede geral de esgotamento sanitário.

b) Perfil extremo 2 (Piores condições de saúde de acordo com a perspectiva dos ODM)

É o perfil da capital que possui:

- Altas taxas de mortalidade infantil;

- Baixa proporção de gestantes que realizaram sete ou mais consultas no pré-natal;

- Baixa taxa de incidência de HIV/Aids;

- Alta taxa de mortalidade por tuberculose respiratória;

- Baixa proporção da população com acesso à rede geral de água;

- Baixa proporção da população com acesso à rede geral de esgotamento sanitário.

Como as capitais se posicionam em relação a cada um desses perfis

Com base nos estudos de Sawyer et al (2000), para verificar como as capitais brasileiras se posicionam em relação a cada um dos perfis, por meio dos escores de GoM, utiliza-se a seguinte classificação para a predominância das características de um perfil:

a) $g_{i k}>=0,75$ em que $k=1$ : capital tipo predominante do perfil 1 (pois pertence com maior intensidade a esse perfil);

b) $g_{i k}>=0,75$ em que $k=2$ : capital tipo predominante do perfil 2 (pois pertence com maior intensidade a esse perfil);

c) $\left(0,55<=g_{i 1}<=0,74\right) \cap\left(0,25<=g_{i 2}\right.$ $<=0,54)$ : capital do tipo misto com predominância do perfil 1 sobre o perfil 2;

d) $\left(0,55<=g_{i 2}<=0,74\right) \cap(0,25<=$ $\left.g_{i 1}<=0,54\right)$ : capital do tipo misto com predominância do perfil 2 sobre o perfil 1;

e) Capitais que não se enquadram em nenhuma das classificações acima foram consideradas "amorfas", ou seja, capitais equidistantes dos perfis extremos e que, portanto, não possuem características que as aproximem de nenhum perfil.

A Tabela 2 mostra a distribuição das capitais brasileiras de acordo com a tipologia descrita. Pode-se observar que aproximadamente $56 \%$ das capitais têm características predominantes de um dos perfis extremos. Considerando as capitais de um desses perfis, as referentes ao perfil 1

\section{Tabela 2: Distribuição das capitais, segundo a predominância de perfis}

\begin{tabular}{|c|c|c|c|}
\hline Perfis Extremos & Predominância do Perfil & Frequência & Percentual \\
\hline & $\operatorname{Amorfa}(\mathrm{A})$ & 1 & 3,70 \\
\hline & Total & 1 & 3,70 \\
\hline \multirow{4}{*}{$\begin{array}{l}\text { Perfil Extremo 1: } \\
\text { Melhores condições de saúde segundo } \\
\text { a perspectiva dos ODM }\end{array}$} & Predominante 1 (P1) & 11 & 40,74 \\
\hline & Misto 1 com 2 (M12) & 6 & 22,22 \\
\hline & Total & 17 & 62,96 \\
\hline & & & \\
\hline \multirow{4}{*}{$\begin{array}{l}\text { Perfil Extremo 2: } \\
\text { Piores condições de saúde segundo a } \\
\text { perspectiva dos ODM }\end{array}$} & Predominante $2(\mathrm{P} 2)$ & 4 & 14,81 \\
\hline & Misto 2 com 1 (M21) & 5 & 18,52 \\
\hline & Total & 9 & 33,33 \\
\hline & Total & 27 & 100,00 \\
\hline
\end{tabular}

Fonte: Elaborada pelos autores 
$(40,74 \%)$ são as mais frequentes, seguidas pelas capitais predominantemente do perfil $2(14,81 \%)$.

Conforme a Tabela 3, em todas as capitais das regiões Sul, Sudeste e CentroOeste, as condições de saúde foram caracterizadas como as melhores entre as capitais brasileiras. Três das quatro capitais com as piores condições de saúde (Porto
Velho, Rio Branco, Macapá e Maceió) pertencem à Região Norte, sendo Maceió a única capital da Região Nordeste a apresentar piores condições de saúde.

Pode-se observar que somente as capitais do Nordeste e Norte foram classificadas como pertencentes aos perfis mistos. A predominância de melhores condições sobre piores condições de saúde

Tabela 3: Capitais brasileiras e respectivos perfis para as condições de saúde de acordo com a perspectiva dos ODM

\begin{tabular}{|c|c|c|c|}
\hline Capital & $\mathrm{g}_{\mathrm{i} 1}$ & $g_{i 2}$ & Perfil \\
\hline Salvador & 0,499 & 0,501 & A \\
\hline Porto Velho & 0,000 & 1,000 & $\mathrm{P} 2$ \\
\hline Rio Branco & 0,000 & 1,000 & $\mathrm{P} 2$ \\
\hline Fortaleza & 0,332 & 0,668 & M21 \\
\hline Macapá & 0,000 & 1,000 & P2 \\
\hline Belém & 0,332 & 0,668 & M21 \\
\hline Aracaju & 0,649 & 0,351 & M12 \\
\hline Maceió & 0,206 & 0,794 & P2 \\
\hline Manaus & 0,332 & 0,668 & $\mathrm{M} 21$ \\
\hline Teresina & 0,588 & 0,412 & M12 \\
\hline São Luiz & 0,588 & 0,412 & M12 \\
\hline Recife & 0,588 & 0,412 & M12 \\
\hline Natal & 0,649 & 0,352 & M12 \\
\hline Boa Vista & 0,649 & 0,352 & M12 \\
\hline João Pessoa & 0,424 & 0,576 & M21 \\
\hline Palmas & 0,424 & 0,576 & M21 \\
\hline Cuiabá & 1,000 & 0,000 & P1 \\
\hline São Paulo & 1,000 & 0,000 & P1 \\
\hline Rio de Janeiro & 1,000 & 0,000 & P1 \\
\hline Porto Alegre & 1,000 & 0,000 & P1 \\
\hline Campo Grande & 1,000 & 0,000 & P1 \\
\hline Florianópolis & 1,000 & 0,000 & $\mathrm{P} 1$ \\
\hline Vitória & 1,000 & 0,000 & P1 \\
\hline Goiânia & 1,000 & 0,000 & P1 \\
\hline Curitiba & 1,000 & 0,000 & $\mathrm{P} 1$ \\
\hline Belo Horizonte & 1,000 & 0,000 & P1 \\
\hline Brasília & 1,000 & 0,000 & P1 \\
\hline
\end{tabular}

Fonte: Elaborada pelos autores 
é mais frequente em capitais do Nordeste. A predominância de piores condições de saúde pode ser observada com maior frequência em capitais do Norte. As capitais que apresentaram perfil de saúde misto com predominância de piores sobre melhores condições são: Manaus, Belém, Fortaleza, João Pessoa e Palmas. Por sua vez, as capitais Aracaju, Teresina, São Luiz, Recife, Natal e Boa Vista pertencem ao perfil misto, com predominância de melhores sobre piores condições de saúde, sendo Boa Vista a única capital da Região Norte incluída nesse perfil. Salvador foi a única capital brasileira não classificada em nenhum dos perfis, sendo considerada amorfa, ou seja, equidistante dos perfis extremos e, portanto, não possuindo características que a aproximem de nenhum perfil.

\section{Conclusões}

O uso de indicadores sociais na avaliação das políticas públicas tem experimentado crescimento substancial, principalmente nas décadas mais recentes, em que se intensificou a necessidade de aperfeiçoar os mecanismos de planejamento e de controle da gestão pública em todas as esferas de governo. Em nível mundial, a ONU tem sugerido o uso de indicadores sociais no acompanhamento da evolução dos países, no que se refere ao alcance das metas propostas no projeto denominado Objetivos de Desenvolvimento do Milênio.

Dois perfis extremos foram delineados para as condições de saúde nas capitais brasileiras: o perfil extremo de melhores condições e o perfil extremo de piores condições de saúde sob a perspectiva dos ODM. O perfil extremo das melhores condições foi caracterizado por: taxas intermediárias de mortalidade infantil, alta proporção de gestantes que realizaram sete ou mais consultas no pré-natal, alta taxa de incidência de HIV/Aids, baixa taxa de mortalidade por tuberculose respiratória, alta proporção da população com acesso à rede geral de abastecimento de água, média e alta proporção da população com acesso à rede geral de esgotamento sanitário. A taxa de incidência de HIV/Aids apresentou resultado, a princípio, inesperado no delineamento dos perfis extremos. Contudo, uma maior incidência dessa doença nas regiões Sul e Sudeste, por exemplo, pode ser explicada, em parte, por atrasos menores nas notificações, quando comparados aos verificados em regiões como o Norte e o Nordeste.

As melhores condições de saúde podem ser encontradas nas capitais dos estados pertencentes às regiões Sul, Sudeste e Centro-Oeste. Enquanto as piores condições de saúde estão presentes em algumas capitais das regiões Norte e Nordeste: Porto Velho, Rio Branco, Macapá e Maceió. Em face desses resultados, pode-se dizer que as condições de saúde nas capitais brasileiras apresentam forte viés regional, apesar da presença de condições mistas em algumas capitais das regiões Norte e Nordeste.

Diante dessas considerações, vale destacar algumas limitações do trabalho. Em primeiro lugar, não foi pretensão deste artigo esgotar as características que podem compor um perfil de melhores condições de saúde, porque não se encontram disponíveis para as capitais brasileiras todos os indicadores sugeridos pela ONU para o acompanhamento do alcance das metas dos ODM relacionadas à saúde. Em segundo lugar, é notória certa "disparidade" regional na qualidade dos dados disponíveis no Brasil, o que pode resultar em "falsas" interpretações.

Como sugestão para estudos futuros, pode-se apontar a extensão do trabalho 
para cidades do interior do "grande sertão chamado Brasil”. São cidades que incontestavelmente vivem uma realidade muito distinta das capitais brasileiras e que, não raramente, sofrem de certo descaso do poder público. Assim, também seria interessante a condução de estudos comparativos, ao longo do tempo e entre os diferentes locais, a fim de propor melhores políticas de destinação de recursos e ações mais focadas na realidade de cada região.

Por fim, é importante lembrar que além de subsidiar os gestores públicos e a sociedade na avaliação das políticas públicas, espera-se que este artigo possa contribuir com o estudo incipiente dos ODM na experiência das capitais brasileiras e com a identificação de sistema de monitoramento das condições de saúde, que seja mais apurado na aferição da eficiência e eficácia das políticas sociais de saúde. Afirmar que os indicadores utilizados na composição dos perfis parecem ter uma função importante na administração da saúde pública é necessário. Essa afirmação faz sentido especialmente quando se considera que os indicadores têm papel norteador para os gestores públicos, uma vez que avaliar a gestão é essencial para corrigir rumos indesejados que podem estar sendo tomados despercebida ou inadvertidamente. Além disso, avaliações periódicas permitem identificar e aproveitar oportunidades de ação para se antecipar ou solucionar problemas, reduzir desperdícios ou realizar ações para atingir compromissos assumidos. Os indicadores também assumem o papel de divulgar as informações e melhorar a comunicação do governo com a sociedade civil.

É possível dizer que a grande pergunta que permeou e motivou o trabalho começa a ser respondida: os ODM parecem contribuir, pelo menos, com o estabelecimento de metas para a formulação e avaliação de políticas públicas, uma vez que podem servir de balizamento para os gestores públicos que, muitas vezes, encontram dificuldades em fixar padrões de resultados em suas gestões. Afinal, como já dizia um velho ditado chinês: "Para quem não sabe onde chegar, qualquer ponto é destino".

(Artigo recebido em janeiro de 2009. Versão final em junho de 2009).

\section{Referências bibliográficas}

Andrade, J. A.; Ussan, J. L. M; Klering, L. R. A Avaliação de Políticas Públicas em Análise: o caso do Sistema Nacional de Avaliação da Educação Básica - Saeb. In: Encontro Anual da Associação Nacional dos Programas de Pós-graduação em Administração, 25 Anais... Campinas: Anpad, 2001.

Arretche, M. T. Tendências no Estudo sobre Avaliação. In: Rıco, Elizabeth Melo (org.) Avaliação de Políticas Sociais: uma questão em debate. São Paulo: Cortez/Iee, 1998.

Banco de Dados do Sistema Único de SAÚde (Datasus). Informações de Saúde: Indicadores de Saúde, Estatísticas vitais, Demográficas e socioeconômicas. Disponível em: http://w3.datasus.gov.br/datasus/datasus.php. Acessado em: 10/Jun/2008. 
Berkman, L. et al. Black/White Differences in Health Status Among the Elderly. Demography. v. 26, n. 4, p. 661-678, nov. 1989.

Ceneviva, R; Farah, M. F. S. Democracia, Avaliação e Accountability: a avaliação de políticas públicas como instrumento de controle democrático. In: II Encontro de Administração Pública e Governança, Anais... São Paulo: EnAPG, 2006.

Fundo das Nações Unidas para a Infância (Unicef). Situação Mundial da Infância 2008. Caderno Brasil. Fundo das Nações Unidas para a Infância, 2008.

Guimarães, J.R.S; Jannuzzi, P.M. Indicadores sintéticos no processo de formulação e avaliação de políticas públicas: limites e legitimidades. In: XIV Encontro Nacional de Estudos Populacionais, Anais... Caxambu: ABEP, 2004.

Instituto de Pesquisa Econômica Aplicada (Ipea). Objetivos de Desenvolvimento do Milênio. Relatório Nacional de Acompanhamento. Coordenação: Instituto de Pesquisa Econômica Aplicada. Brasília: Ipea, 2005.

- Objetivos de Desenvolvimento do Milênio. Relatório Nacional de Acompanhamento. Coordenação: Instituto de Pesquisa Econômica Aplicada e Secretaria de Planejamento e Investimentos Estratégicos. Brasília: Ipea: MP, SPI, 2007.

JannuZzi, P.M. Considerações sobre o uso, mau uso e abuso dos indicadores sociais na formulação e avaliação de políticas públicas municipais. Revista de Administração Pública, Rio de Janeiro, v. 36, n. 1, p. 51-72, jan.-fev. 2002.

Repensando a prática de uso de indicadores sociais na formulação e avaliação de políticas públicas municipais. In: Encontro Anual da Associação Nacional dos Programas de Pós-graduação em Administração, 25 Anais... Campinas: Anpad, 2001a. . Indicadores sociais no Brasil: conceitos, fontes e aplicações. Campinas: Alínea/

PUC-Campinas, 2001b.

Machado, C. J. Perfis de Morbi-Mortalidade Infantil no Estado de São Paulo, 1994: uma aplicação de grade of membership à análise de causas múltiplas de morte. Belo Horizonte: Cedeplar/UFMG, 1997. (Dissertação de Mestrado).

Manton, K. G.; Woodbury, M. AA.; Tolley, H. D. Statistical Application Using Furzy Sets. New York: John Wiley \& Sons, 1994.

MinISTÉRIO DA SAÚDE. Painel de Indicadores do SUS. Secretaria de Gestão Estratégica e Participativa. Departamento de Monitoramento e Avaliação da Gestão do SUS. Brasilia, 2006.

Organização Mundial de Saúde (OMS). Catalogue of health indicators. Genebra, 1996. Disponível em: http://whqlibdoc.who.int/hq/1996/WHO_HST_SCI_96.8.pdf. Acessado em: 15 de jun. 2008.

ORGANIZAÇÃO DAS NAÇÕES UNIDAS (ONU). Indicators for Monitoring the Millennium Development Goals: definitions, rationale, concepts and sources. New York: United Nations, 2003.

OrganizaÇão PAN-Americana da SAúde (Opas). Indicadores básicos para a saúde no Brasil: conceitos e aplicações. Rede Interagencial de Informação para a Saúde (Ripsa), 2. ed. Brasília: Opas, 2008.

Pereira, M. G. Epidemiologia: Teoria e Prática. Rio de Janeiro: Guanabara-Koogan, 1995.

Programa das Nações Unidas para o Desenvolvimento (PNUD). Desenvolvimento humano e condições de vida: indicadores brasileiros. Brasília: PNUD, 1998. 
Sawyer, D. O.; Fígoli, M. G. B.; Rodrigues, R. N.; Garcia, R. A. Caracterização dos Tipos de Doadores de Sangue em Belo Horizonte: Heterogeneidade do Homogêneo. In: XII Encontro de Estudos Populacionais. Anais..., Caxambu: Abep, 2000.

Silva, P. L. B.; Melo, M. A. B. O processo de implementação de políticas públicas no Brasil: características e determinantes da avaliação de programas e projetos. Núcleo de Estudos de Políticas Públicas - NEPP. Caderno no 48. Campinas, 2000.

Sawyer, D. O.; Leite, I. C.; Garcia, R. A. Perfis de Utilização de Serviços de Saúde no Brasil. Ciência \& Saúde Coletiva, v. 7, n. 4, p. 757-776, 2002.

Sistema Nacional de Informações de Saneamento (SNIS). Água e Esgoto. Disponível em: http://www.snis.gov.br Acessado em: 12 de jun. de 2008.

TAm, N. P.; Borges, A. A segmentação de mercado a partir do Método Fuzzy Logic: uma alternativa metodológica para o problema de superposição dos segmentos na classificação. In: Encontro Anual da Associação Nacional dos Programas de Pósgraduação em Administração, 26 Anais... Salvador: Anpad, 2002.

Torres, H. G.; Ferreira, M. P.; Dini, N. P. Indicadores sociais: por que construir novos indicadores como o IPRS. São Paulo em Perspectiva, v. 17, n. 3-4, jul-dez 2003. 


\section{Resumo-Resumen-Abstract}

Perfil das condições de saúde das capitais brasileiras na perspectiva dos Objetivos de Desenvolvimento do Milênio (ODM)

Danielle Ramos de Miranda Pereira, Marcelo de Rezende Pinto, Álida Rosária Silva Ferreira e Rodrigo Nunes Ferreira

No contexto atual, o uso de indicadores sociais na avaliação das políticas públicas tem-se tornado cada vez maior. Os indicadores sociais também têm sido implementados no acompanhamento dos Objetivos de Desenvolvimento do Milênio (ODM), projeto da Organização das Nações Unidas $(\mathrm{ONU})$ em que vários países, inclusive o Brasil, firmaram um pacto para tornar o mundo menos desigual. Sendo assim, para avaliar o cumprimento das metas estabelecidas nos ODM, este artigo pretende delinear perfis das capitais brasileiras quanto às condições de saúde, sob a perspectiva dos ODM, a fim de contribuir para a formulação e avaliação de políticas públicas e indicar um caminho para a escolha de indicadores importantes de serem monitorados. Foram delineados perfis, com a adoção do método Grade of Membership, quanto à saúde nas capitais brasileiras. Perante os resultados obtidos, pode-se dizer que as condições de saúde nas capitais brasileiras apresentam forte viés regional.

Palavras-chave: Objetivos de Desenvolvimento do Milênio, condições de saúde, formulação e avaliação de políticas públicas

Perfil de las condiciones de la salud de las ciudades brasileñas, en la perspectiva de los Objetivos de Desarrollo del Milenio (ODM)

Danielle Ramos de Miranda Pereira, Marcelo de Rezende Pinto, Álida Rosária Silva Ferreira y Rodrigo Nunes Ferreira

En el contexto actual, el uso de indicadores sociales en la evaluación de las políticas públicas ha ascendido. Los indicadores sociales también han sido aplicados en la vigilancia de la Metas de Desarrollo del Milenio (ODM), un proyecto de las Naciones Unidas (ONU) en el que varios países, entre ellos Brasil, han firmado un pacto para que el mundo sea menos desigual. Por lo tanto, para evaluar el cumplimiento de los objetivos fijados en los ODM, este artículo intenta esbozar los perfiles de las capitales brasileñas en materia de salud, desde la perspectiva de los ODM, a fin de contribuir a la formulación y evaluación de políticas públicas y orientar en la selección de indicadores importantes a ser monitorizados. Se esbozaron los perfiles con la adopción del método de Grade of Membership, respecto a la salud en las capitales brasileñas. Ante los resultados, podemos decir que la salud en las ciudades brasileñas tiene un fuerte sesgo regional.

Palabras clave: Objetivos de Desarrollo del Milenio, las condiciones de salud, la formulación y evaluación de políticas públicas

Health conditions at Brazilian state capitals from the perspective of the Millennium Development Goals (MDG)

Danielle Ramos de Miranda Pereira, Marcelo de Rezende Pinto, Alida Rosária Silva Ferreira and Rodrigo Nunes Ferreira

In the current context, the use of indicators in the evaluation of public policies has become greater. Indicators have been implemented in monitoring the Millennium Development Goals (MDG), project of the United Nations (UN) in which countries signed a pact to make the world less unequal. Therefore, using the indicators suggested by the UN to assess compliance with the goals set in the MDG, this article aims to outline profiles of Brazilian capital cities on health, from the 
perspective of the millennium goals, to contribute to the formulation and evaluation of public policies to give direction on the choice of key indicators to be monitored. Profiles have been outlined, with adoption of the method Grade of Membership, for best, worst and mixed heath conditions in Brazilian capitals. With the results, we can say that health conditions in these capitals have a strong regional bias.

Keywords: Millennium Development Goals, health conditions, formulation and evaluation of public policies.

Danielle Ramos de Miranda Pereira

Doutora em Demografia pelo Cedeplar/UFMG. Atualmente é gestora de Educação e Pesquisa da Fundação João Pinheiro (FJP/MG).

Contato: daniellermp@yahoo.com.br

Marcelo de Rezende Pinto

Doutorando em Administração pelo Cepead/UFMG. É professor do Departamento de Administração da Pontifícia Universidade Católica de Minas Gerais (PUC/Minas).

Contato:marcrez@hotmail.com

Álida Rosária Silva Ferreira

Mestre em Demografia pelo Cedeplar/UFMG. Trabalha na Secretaria de Planejamento, Orçamento e Informação da Prefeitura de Belo Horizonte.

Contato: alida_ferreira@yahoo.com.br

Rodrigo Nunes Ferreira

Mestre em Geografia pela UFMG. Trabalha na Secretaria de Planejamento, Orçamento e Informação da Prefeitura de Belo Horizonte.

Contato: rnf60@yahoo.com.br 